\title{
Electrodynamics of the equatorial F-region ionosphere during pre-sunrise period
}

\author{
S. R. Prabhakaran Nayar ${ }^{1}$, T. J. Mathew ${ }^{1}$, C. V. Sreehari ${ }^{1}$, S. G. Sumod ${ }^{1}$, C. V. Devasia ${ }^{2}$, S. Ravindran ${ }^{2}$, V. Sreeja ${ }^{2}$, \\ T. Kumar Pant ${ }^{2}$, and R. Sridharan ${ }^{2}$ \\ ${ }^{1}$ Department of Physics, University of Kerala, Kariavattom, Trivandrum, 695 581, India \\ ${ }^{2}$ Space Physics Laboratory, Vikram Sarabhai Space Centre, Trivandrum 695 022, India
}

Received: 10 July 2008 - Revised: 18 November 2008 - Accepted: 4 December 2008 - Published: 6 January 2009

\begin{abstract}
The electrodynamics of the pre-sunrise equatorial F-region is investigated using HF Doppler radar and digital ionosonde. The observations are limited to those days for which the radar probing frequency is below the $f o \mathrm{~F} 2$ value. The ionosphere observation using HF Doppler radar exhibit interesting features during pre-sunrise period similar to the post sunset pre-reversal enhancement. The most striking feature observed during pre-sunrise period is the sudden downward excursion in the vertical drift around local sunrise followed by an upward turning. Pre-sunrise observations of vertical plasma drift and the sunrise downward excursion followed by an upward turning after the ground sunrise related to the zonal electric field at the equatorial F-region are the most significant results not reported earlier.
\end{abstract}

Keywords. Ionosphere (Electric fields and currents; Equatorial ionosphere; Ionospheric disturbances)

\section{Introduction}

Vertical plasma drift at the equatorial F-region during postsunset and pre-sunrise period is primarily driven by the zonal electric field formed as part of the polarization field developed due to thermospheric wind and the sharp conductivity gradients in the E-region. Though the characteristics of the post sunset enhancement in vertical plasma motion has been extensively studied, the pre-sunrise phenomena is not yet studied in detail. At the equatorial F-region, around the sunrise and sunset, the pressure gradients in the neutral air produce zonal winds which give rise to vertical currents and polarization fields on both sides of the terminators (Rishbeth, 1971; Farley et al., 1986; Eccles, 1998). The electric field created by the F-region dynamo effect maps to the magnetically conjugate E-region connected to the equatorial F-region by the equipotential magnetic field lines. When the E-layer is sunlit, it almost entirely short circuits the F-layer polarization fields (Rishbeth, 1971). The enhancement in vertical drift during the post sunset period is explained in terms of the polarization fields produced by the large westward gradient of the ionospheric conductivity across the evening terminator. Around the sunrise, the F-region electric field builds first for a short duration and then the E-region conductivity starts increasing resulting in the vertical velocity pattern due to the added effect of E- and F-regions. This type of horizontal gradient in the ionospheric conductivity should exist near the morning sunrise terminator leading to similar anomalous feature in vertical drift.

Most of the reviews of the zonal electric field (vertical drift) variability in the equatorial ionospheric F-region tend to ignore electric field perturbations and equatorial anomaly near the sunrise, although it was clearly visible in studies from the equatorial Jicamarca radar site where it was seen at times as an enhancement in the zonal electric field (Aggson et al., 1995). The main difficulty in probing the presunrise ionosphere using ground based radar system is the reduced electron density prevailing near sunrise. A multi frequency HF Doppler radar located at the magnetic equatorial station, Trivandrum $\left(8.33^{\circ} \mathrm{N}, 77^{\circ} \mathrm{E}\right.$, dip $\left.0.4^{\circ} \mathrm{N}\right)$, operating at three fixed frequencies $2.5 \mathrm{MHz}, 3.5 \mathrm{MHz}$ and $4.5 \mathrm{MHz}$, is capable of observing the characteristics of vertical plasma motion at three altitudes. In this work, the vertical velocity profile during morning hours are presented and their features are discussed in terms of F- and E-region dynamos and are compared with the post sunset behavior of vertical plasma motion.

Correspondence to:

S. R. Prabhakaran Nayar

(srpnayar@gmail.com)

Published by Copernicus Publications on behalf of the European Geosciences Union. 


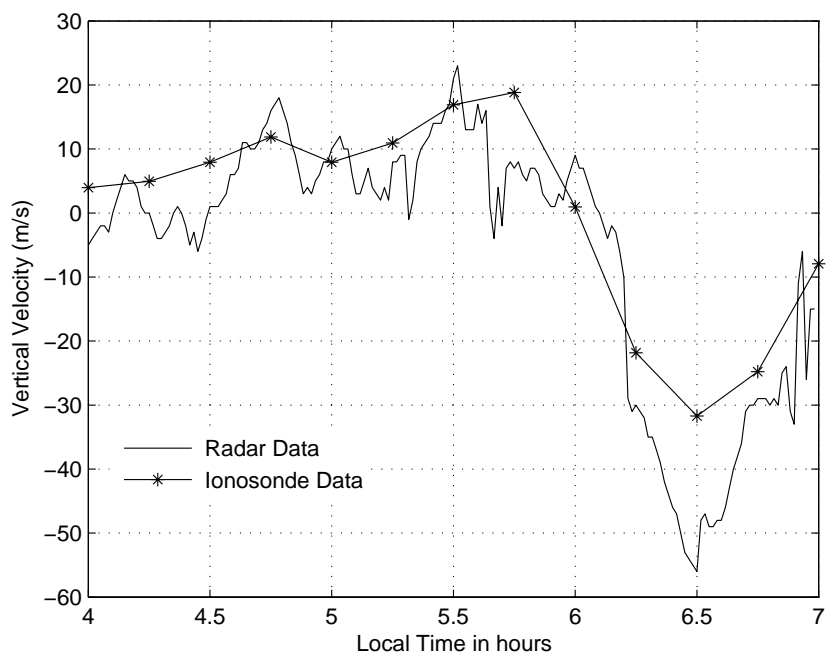

Fig. 1. F-region vertical velocity profile of 23 December 2004, observed at $2.5 \mathrm{MHz}$ using both HF Doppler radar (one minute resolution) and ionosonde (15 min resolution). Ground sunrise time 06:32 LT

\section{Experiment}

The Multi-frequency HF Doppler radar installed at the University of Kerala is a coherent mono-static pulsed system capable of quadrature detection of ionospheric echoes (Nayar and Sreehari, 2004). It uses a single broadband transmitter and works with three fixed frequencies $-2.5 \mathrm{MHz}, 3.5 \mathrm{MHz}$ and $4.5 \mathrm{MHz}$. For the vertical sounding, the transmitted signal is reflected back from the ionosphere and is received by a three element folded type dipole antenna capable of wide band operation. The received signals are fed to the phase coherent receivers and the resulting quadrature outputs are fed to the digital data acquisition system for recording the high resolution Doppler data. In the case of single frequency operation, at every one minutes, the vertical drift is evaluated using the time series of Doppler data. For multi-frequency operation, a sequential switching scheme is followed and the data resolution is six minutes. To complement the $\mathrm{HF}$ Doppler radar observations, the digital ionosonde observation from Space Physics Laboratory, VSSC, Trivandrum is used to evaluate the vertical plasma motion from the time variations of $h^{\prime} F$ during the pre-sunrise period.

The plasma drift observed by HF Doppler radar during the morning hours are apparent as the observed values includes the contribution due to production of ions. The production contribution to the observed vertical drift is to be considered if the reflection height is below $300 \mathrm{~km}$ (Bittencourt and Abdu, 1981). Bittencourt and Abdu (1981) have shown that, for equatorial stations, the rate of change of the $h^{\prime} F$ is indicative of the variations of F-region vertical plasma drift and thus the zonal electric field when $h^{\prime} F$ is greater than about $300 \mathrm{~km}$. Even for the lower altitudes of the $h^{\prime} F$, the rapid change of $h^{\prime} F$ generally indicates electric field perturbation. The correction in the vertical drift due to the production is required only when the reflection altitude is below $300 \mathrm{~km}$. Since the group retardation effect due to underlying ionisation would be very small for the value of $h^{\prime} F$ at $2.5 \mathrm{MHz}$, it can be taken as the true height for the present study.

However, to account for the vertical velocity term introduced due to the production, a correction scheme is applied to the measured vertical drift. In the F-region (above $150 \mathrm{~km}$ ) the dominant ions formed by solar irradiance are $\mathrm{O}^{+}$ions; the radiation absorbed by the molecular nitrogen does not contribute appreciably to the observed ionization, because molecular ions are short lived in the F-region (Rishbeth and Setty, 1961). So $\mathrm{O}^{+}$ions are produced in the F-region altitude through the reaction: $\mathrm{N}^{+}+\mathrm{O}_{2} \rightarrow{ }^{k} \mathrm{O}^{+}+\mathrm{NO}$, Where $k=2 \times 10^{-11} \mathrm{~cm}^{3} \mathrm{~s}^{-1}$ as given by Torr and Torr (1979). The production coefficient " $\rho$ " in the lower altitude is defined as $\rho=k n\left[\mathrm{O}_{2}\right]$. Where $n\left[\mathrm{O}_{2}\right]$ is the is the concentration of $\mathrm{O}_{2}$, which is obtained from MSIS model. Now, the apparent drift due to production is $V=\rho H$, Where $H=\left(N^{-1} d N / d h\right)^{-1}$ is the ionization scale height, where $N$ is the electron density, and $h$ is the height and the value of ionization scale length $(H)$ at the F-region during morning hours between 2 and $2.5 \mathrm{MHz}$ is taken as $10 \mathrm{~km}$. The scale length is expected to vary with time and with solar conditions. Since the probing altitudes for the selected operational frequencies are close by, evaluation of the height gradient in vertical drift may not be significantly affected by the variation in $H$. The movement of the reflection level of the F-region (at the magnetic equator) is controlled by the electrodynamic drift $(\boldsymbol{E} \times \boldsymbol{B})$ and the apparent drift due to production. But the effect of production is dominant at lower altitude and becomes insignificant at higher altitudes. The apparent drift due to production is calculated for different altitudes around the region of the observation and it is found that the correction due to the production is less than $10 \mathrm{~ms}^{-1}$ implying that the large downward drift observed near sunrise is due to the $\boldsymbol{E} \times \boldsymbol{B}$ drift.

\section{Observation}

Figure 1 presents the vertical drift observed using the HF Doppler radar operated at $2.5 \mathrm{MHz}$ on 23 December 2004 during 04:00 to 07:00 LT. For comparison, the vertical drift determined from the ionosonde at $2.5 \mathrm{MHz}$ is also presented in Fig. 1. The difference in magnitude of the drift between them is due to the different time and height resolutions used in the two instruments. Since the ionograms are recorded generally at $15 \mathrm{~min}$ intervals, the resolution of the vertical drift using ionosonde is coarsed compared to that obtained using HF Doppler radar. Moreover, the velocity observed using HF Doppler radar is calculated from the Doppler shift, but in the case of ionosonde, it is calculated from the variation of virtual heights (large difference in velocity observed near sunrise). The profile reveals many interesting features 

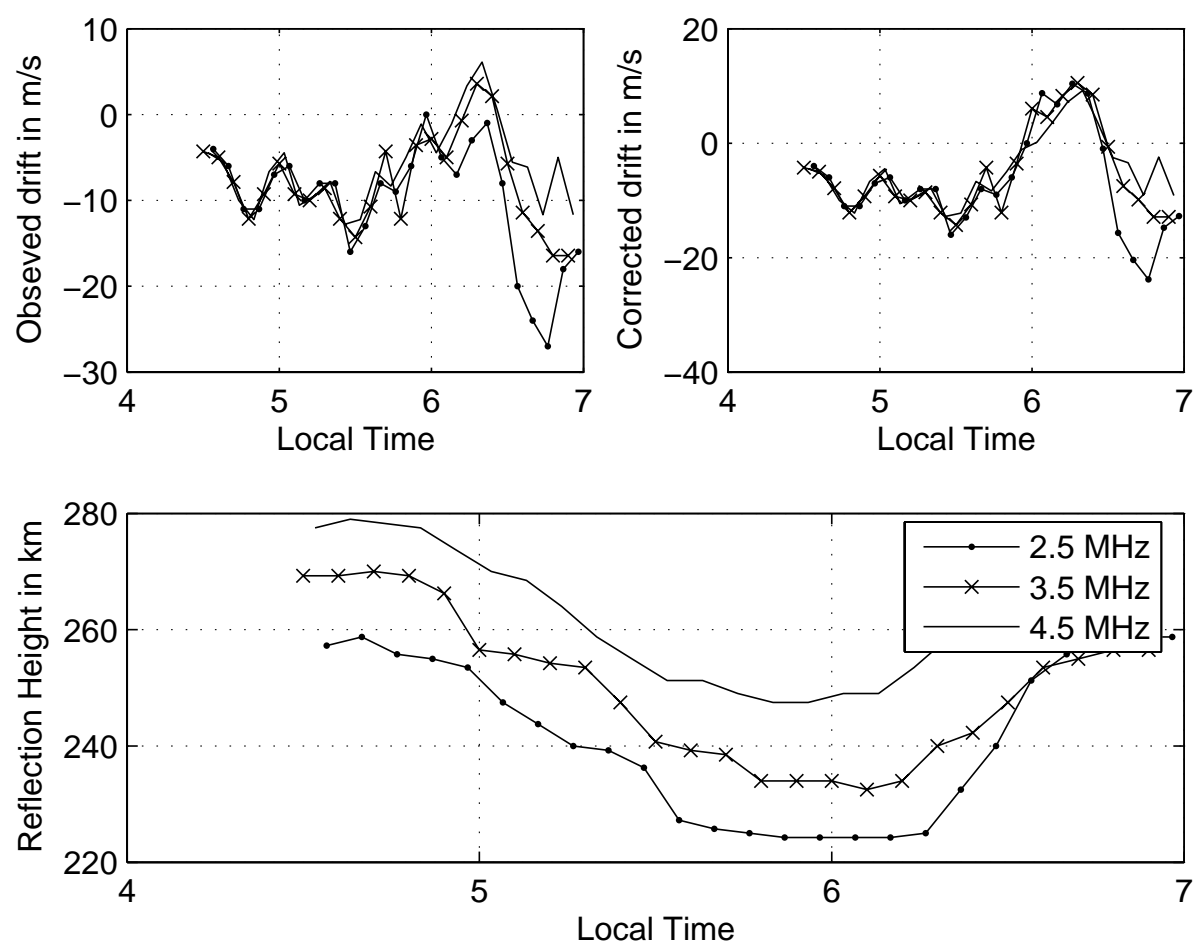

Fig. 2. The observed (top left panel) and corrected (top right panel) multi-frequency vertical velocity profile on 19 March 2004 . Variation of the reflection height is shown in the bottom panel. Ground sunrise time - 06:27 LT.

of equatorial vertical plasma drift near sunrise. The ground sunrise on this day is at 06:32 LT. Sunrise at the probing altitude $(300 \mathrm{~km})$ is at $05: 23 \mathrm{LT}$ where the F-region starts build up. The E-region sunrise on this day is around 05:50 LT where the downward enhancement starts. The most important feature in Fig. 1 is the downward excursion of the vertical plasma drift for about half an hour around sunrise (06:00-06:30 LT). During this period, since the altitude of observation is above $300 \mathrm{~km}$, the correction due to production is not significant (Bittencourt and Abdu, 1981). The vertical drift remains positive (upward) during early morning periods with few oscillations and then suddenly turns downwards at around 06:00 LT and decreases to about $-48 \mathrm{~ms}^{-1}$ around 06:30 LT. Then the drift turns upwards and gradually increases to the normal daytime value. This feature is present on all the days for which the observations could be made. Further, significant day-to-day variations in the magnitude of the downward maximum and the duration of the enhancement is also observed. Another important feature of the plasma drift is the upward enhancement of the plasma drift just prior to its downward excursion.

The pre-sunrise period observation of the ionosphere is possible for the days for which the electron concentration is sufficient for the operation of $2.5 \mathrm{MHz}$. As mentioned in the last section, for most of the days, the minimum value of $f o \mathrm{~F} 2$ lies below the probing frequency of the radar in the pre sunrise hours. However, 19 March 2004 was an exceptional day, for this day, the minimum value of $f o \mathrm{~F} 2$ remained high, making the operation of all the three frequencies of the HF Doppler radar possible. The observed vertical velocity profile (top left), the corrected drift (top right) and the corresponding observation altitudes (bottom) on 19 March 2004 during morning hours between 04:00 LT and 07:00 LT at three frequencies $2.5 \mathrm{MHz}, 3.5 \mathrm{MHz}$ and $4.5 \mathrm{MHz}$ are as shown in Fig. 2.

As the observed drift is the sum of electrodynamic drift and apparent drift due to production, to extract the electrodynamic drift, the contribution due to $\rho H$ at every reflection height is calculated and is removed from the observed drift from 06:00 LT onwards (about half an hour prior to ground sunrise, at which the the value of $f_{o} \mathrm{~F} 2$ starts increasing). The variation in the reflection heights of the three frequencies and the corresponding plasma drifts suggests the possible existence of the height gradient and velocity vortex during the pre-sunrise period similar to the one existing in the postsunset period (Nayar and Sreehari, 2004). The vertical drifts show an upward tendency from 05:30LT onwards, reach a small maxima around 06:20 LT and then start decreasing. The local ground sunrise on this day was at 06:27 LT and the sunrise time at the probing altitude ranges from 05:24 to $05: 27$ LT. The sudden downward enhancement (SDE) has started around $20 \mathrm{~min}$ before the ground sunrise. After the 
sunrise, the profile attains the day time behavior. Overall, the SDE followed by the upward turning appears to be consistent signature of the vertical velocity profile near sunrise, where a small but identifiable upward enhancement prior to ground sunrise is often observed.

\section{Results and discussion}

The downward enhancement of vertical plasma drift, as seen from Fig. 2, is a clear observed phenomenon during sunrise at which many physical processes like photo-ionization, chemical loss, diffusion, electrodynamic drift, coupling between E- and F-regions of the ionosphere etc come together into action. It is really difficult to isolate the contribution due to each of these processes. Crain et al. (1993), based on the theory of Farley (1986) and calculations using DE 2 satellite measurements explained that the pre-reversal enhancement (PRE) is due to the fields produced to prevent negative charge accumulation at E-region sunset terminator. Similarly around sunrise terminator, the plasma polarizes to produce an electric field that is downward, then upward, on each side of sunrise terminator. These electric fields, when mapped to the E-region, produce Hall currents convergent at the terminator boundary. The plasma polarizes, preventing a buildup of positive charge at sunrise terminator, and these polarization fields produce downward, then upward drifts on each side of sunrise terminator. The observed drift profile, downward enhancement followed by upward reversal, agrees with these suggestions pointing to the existence of a morning phenomenon complementary to PRE.

At equatorial latitudes, both the geomagnetic field and the F-region zonal electric field are almost perpendicular to each other. Then, from Maxwell's equations, $\nabla \times \mathbf{E}=-(\partial \mathbf{B} / \partial t)$ and if one assumes local time invariance of geomagnetic field during quiet conditions, $\nabla \times \mathbf{E}=0$, i.e. the electric field should be curl-free or irrotational. The curl-free nature was in fact theoretically suggested by Rishbeth (1971). Near the sunset and sunrise, where the charge density is varying with local time, the vertical polarization electric field lines of force are curved; the plasma drifts being normal to the electric lines of force, therefore converge or diverge in the vertical direction. Rishbeth (1971) modeled $300 \mathrm{~km}$ as the altitude where plasma drift becomes purely horizontal. The morning multiple height vertical drifts (after chemical correction) presented in Fig. 2 also seem to obey the curl-free nature in a curved field line scenario near 06:15-06:45 LT sector.

The morning vertical velocity profiles obtained from multi-frequency HF Doppler radar shows a small pre-sunrise upward enhancement just before sudden downward enhancement (SDE) as modeled by Fareley et al. (1986). Aggson et al. (1995) have reported enhancements of eastward electric fields near sunrise in the equatorial F-region using the data base of San Marco D satellite. Although the sunrise enhancement in the vertical velocity does not occur for ev- ery day in the data set, it is clear from the data base of the HF Doppler radar data that an identifiable increase in the upward drift occurs on most of the days on which observation was possible. These measurements appear to confirm the original Jicamarca study which found an occasional morningside enhancement in the vertical updrafting velocity at sunrise (Aggson et al., 1995). On seasonal averaging of both the Jicamarca data (Fejer, 1991) and the San Marco data, these morning-side enhancements appear to get averaged out and do not appear as a notable feature on the mean. Nicolls et al. (2006) have shown that eastward electric field is not necessary to produce an upward enhancement but sometimes the uplift occurs as the ionospheric response to a decreasing westward electric field in conjunction with sufficient recombination.

During sunrise, the physical processes operative at equatorial F-region are - photo ionization, plasma diffusion, loss processes and the plasma drifts. The upward turning in the vertical plasma drift near ground sunrise is a clear indication of the coupling between the F- and E-regions of the ionosphere. The mechanism of SDE can also be qualitatively explained. The dominance of the F-region dynamo depends on the relative values of the field- integrated E- and Fregion conductivities. During daytime, the F-region dynamo is short circuited by the E-region at conjugate magnetic latitudes. But during F-layer sunrise, F-region dynamo builds up creating a westward electric field and drives a downward drift. Just before ground sunrise, the E-layer gets enough charge density to operate its own dynamo and thereby begins short-circuiting of the F-region dynamo. Thus the downward drift reaches its maximum near the time of ground sunrise and gradually degrades to small daytime upward drift about an hour later. The negative maximum of SDE represents a switch-over time at which the F-region gives the control of equatorial electrodynamics to E-region.

\section{Conclusions}

The vertical velocity profile during morning hours with good data resolution (one minute) over Trivandrum, shows a downward drift (westward electric field) of about 20-30 min duration near the ground sunrise. A small upward enhancement is observed prior to the ground sunrise on many days. But this pre-sunrise enhancement is not a regular feature of the morning behavior of the equatorial ionosphere. The limited data set of multi-frequency profile shows a height gradient near sunrise and thereby satisfies the curl free nature of the electric field. Thus, the equatorial ionospheric F-region vertical velocity profiles selected from the data base of multi frequency HF Doppler radar reveal a motion in the pre sunrise hours which is complementary to the pre-reversal enhancement near sunset.

Acknowledgements. The work is carried out with financial support from ISRO under the RESPOND and CAWSES programmes. 
Topical Editor M. Pinnock thanks one anonymous referee for her/his help in evaluating this paper.

\section{References}

Aggson, T. L., Herrero, F. A., Johnson, J. A., Pfaff, R. F., Lmkso, H., Maynardt, N. C., and Moses, J. J.: Satellite observations of zonal electric fields near sunrise in the equatorial ionosphere, J. Atmos. Terr. Phys., 57, 19-24, 1995.

Bittencourt, J. A. and Abdu, M. A.: A theoretical comparison between apparent vertical velocities and real vertical $E \times B$ plasma drift velocities in the equatorial F-region, J. Geophys. Res., 86(A4), 2451-2454, 1981.

Crain, D. J., Heelis, R. A., and Bailey, G. J.: Effects of electrical coupling on equatorial ionospheric plasma motions: When is the F region a dominant driver in the low-latitude dynamo, J. Geophys. Res., 98(A4), 6033-6037, 1993a.

Crain, D. J., Heelis, R. A., Bailey, G. J., and Richmond, A. D.: Lowlatitude plasma drifts from a simulation of the global atmospheric dynamo, J. Geophys. Res., 98(A4), 6039-6046, 1993 b.

Eccles, J. V.: Modeling investigation of the evening prereversal enhancement of the zonal electric field in the equatorial ionosphere, J. Geophys. Res., 103 26709-26720, 1998.
Farley, D. T., Bonelli, E., Fejer, B. G., and Larsen, M. F.: The prereversal enhancement of the zonal electric field in the equatorial ionosphere, J. Geophys. Res., 91, 13723-13728, 1986.

Fejer, B. G., de Paula, E. R., Gonzales, C. A., Kelley, M. C., and Woodman, R. F.: Average vertical and zonal F-region plasma drifts over Jicamarca, J. Geophys. Res., 96, 13901-13906, 1991.

Nayar, S., Prabhakaran, R., and Sreehari, C. V.: Investigation of height gradient in vertical plasma drift at equatorial ionosphere using multi-frequency HF Doppler radar, J. Geophys. Res., 109, A12308, doi:10.1029/2004JA010641, 2004.

Nicolls, M. J., Kelley, M. C., Valsov, M. N., Sahai, Y., Chau, J., Hysell, D. L., Fagundes, P. R., Becker-Gudes, F., and Lima, W. L. C.: Observation and modeling of post-midnight near the magnetic equator, Ann. Geophys., 24, 1317-1331, 2006, http://www.ann-geophys.net/24/1317/2006/.

Rishbeth, H.: Polarization fields produced by winds in the equatorial F-region, Planet. Space Sci., 19, 357-369, 1971.

Rishbeth, H. and Setty, C. S. G. K.: The F-layer at sunrise, J. Atmos. Terr. Phys., 20, 263-276, 1961.

Torr, D. G. and Torr, M. R.: Chemistry of thermosphere and ionosphere, J. Atmos. Terr. Phys., 41, 797-839, 1979. 